\title{
Astrocytes, Metabolism, Signaling and Brain Drains: Introduction to the Special Issue in Honor of Gerald Dienel
}

Caroline $\operatorname{Rae}^{1} \cdot$ Ursula Sonnewald $^{2}$

Published online: 27 November 2015

(c) Springer Science+Business Media New York 2015

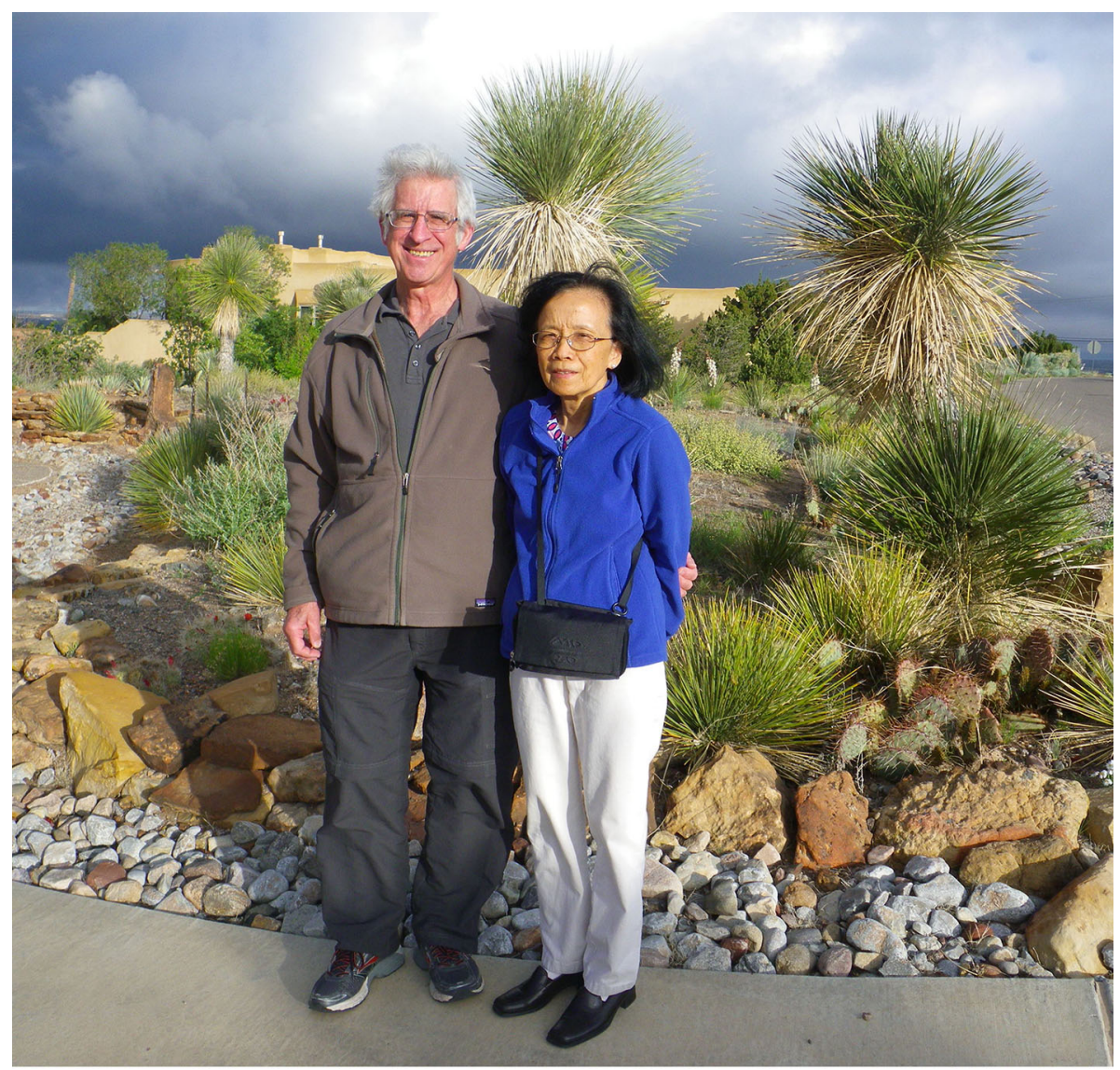

Gerry Dienel and Nancy Cruz, recently in New Mexico.

Caroline Rae

c.rae@neura.edu.au

1 Neuroscience Research Australian \& The University of New South Wales, Randwick, NSW, Australia

2 Norwegian University of Science and Technology (NTNU), Trondheim, Norway 
It is August 2015 and Gerry Dienel is out in the wilderness, climbing mountains and trailblazing, challenging himself and appreciating the beauty inherent in the view along the way. The careful planning and admirable skills that go into making each adventure into the wilderness something special reflect the same attitude of challenge and appreciation that Gerry has applied to his research in neurochemistry.

A graduate of Harvard University, with time out during his university years to serve in the Army Signal Corps in Vietnam, Gerry's formative postdoc years were spent with Thomas Duffy at Cornell Medical School, followed by studies on glucose and deoxyglucose metabolism with Louis Sokoloff at NIH. His work there included the beautiful autoradiography work for which he is rightly well known, including metabolite spreading under activated conditions, work which led him through gap junctions and eventually into the perivascular space.

Gerry is well known for his careful metabolic work, including calculations of relative contributions to metabolism from glucose, glycogen and lactate. This latter molecule is one for which Gerry holds a healthy respect but certainly not awe, understanding, as only one well educated in thermodynamics can, how lactate is a responder rather than a leader. His works on astrocyte activation, done variously with Leif Hertz, Louis Sokoloff and Nancy Cruz are amongst his most highly cited papers; they use systems biochemical thinking to clearly explain the stunning logic of complex metabolism.

This issue of Neurochemical Research is dedicated to Gerry. Contributed to by researchers who are all known to Gerry, it highlights work that that builds upon the foundations of research undertaken by Gerry and also his long term partner in the lab and in life, Nancy Cruz. The issue also highlights astrocytes and their complex roles in brain function and charts the path through discovery that was taken by Gerry in his research career.

The first section of the issue addresses emerging physiological roles of astrocyte activities. It opens with a review of the role of astrocytes as interoceptors in control of metabolic homeostasis, acting as primary detectors of the microenvironment, and/or as signal amplifiers [1]. Microsensing is further examined in a review of redox signaling and control via $\mathrm{NAD}^{+} / \mathrm{NADH}[2]$.

A beautiful piece of work by Amin Derouiche and colleagues (see cover illustration, this issue) showed that fine, peripheral, astrocyte processes contain multiple tiny mitochondria, indicating that ATP generation in these peripheral areas of astrocytes may not be as glycolytic as is currently held.

Three articles on glio-signalling deal with aspects of astrocyte control of homeostasis in the brain using calcium and cyclic AMP among other molecules. These are reviewed in an article submitted by Robert Zorec and further studied in two papers from the NeuroMet group at the University of Copenhagen through original research papers exploring the role of calcium and AMPK in metabolism.

The role of calcium in exocytotic glutamate release from synaptosomes is explored in a comprehensive review on glutamate release from Tetsufumi Ueda's laboratory. This work also provides an historical and theoretical overview of the synapse concept, nicely illustrating the role of the malate-aspartate shuttle and 2-oxoglutarate in generation of synaptic glutamate. Epigenetic modification of glutamate uptake mechanisms in conditions such as alcoholism is then explored in an original research paper showing how distribution of GLAST in astrocytes can be altered by heavy drinking [3].

Gerry's interest in metabolic modelling and glycogen is reflected in two papers from Minnesota. In the first of these, the eternal question of glycogen turnover under steady state conditions is revisited [4]. Gerry and Nancy Cruz in 2002 showed the high sensitivity of brain glycogen levels to experimental conditions and animal handling/stress, as well as high glycogen levels in rats maintained with limited environmental enrichment [5].

Metabolic modelling continues to be a challenge, given the complexities of systems biochemistry and the limited number of variables and constants that can be measured with accuracy. Model choice is a vexed question, as was addressed famously by George Box in stating "Remember that all models are wrong; the practical question is how wrong do they have to be to not be useful?" [6]. In this issue, Pierre-Gilles Henry has attempted to make model choice a little easier for scientists by describing an automated method for modifying and testing models [7].

Gerry and Nancy recently proposed that stimulation of glycogenolysis by the monoaminergic system in locus coeruleus might block glucose uptake by astrocytes in this area [8]. This idea has now been explored more fully in this issue by Mauro DiNuzzo and colleagues.

No issue on metabolic neurochemistry would be complete without mention of ATP. Leif Hertz and Liang Peng, long term collaborators with Gerry, have teamed with Marie Gibbs and colleagues to discuss the role of the $\mathrm{Na}^{+} / \mathrm{K}^{+}$ ATPase in astrocytic control of $\mathrm{K}+$ concentrations and the relative contribution of glycogenolysis, glycolysis and oxidative phosphorylation to supplying the vast amounts of ATP that this enzyme requires. ATP levels are altered by activation of NMDA receptors with effects on mitochondrial $\mathrm{Ca}^{2+}$ levels; the dip in ATP levels is best alleviated by glucose [9]. 
Gerry's early work explored the changes in expression of proteins following cerebral ischemia, including highlighting the vulnerability of proteins with short half-lives, the expression of heat-shock proteins and of ornithine decarboxylase. His work also included ${ }^{45} \mathrm{Ca}$ autoradiography, showing regional spatial and temporal accumulation of calcium in the brain following ischemic injury [10].

This issue includes a number of manuscripts addressing metabolic sequelae that occur after brain injury. The developing brain following injury is reviewed by Mary McKenna and coauthors, while Arthur Cooper and colleagues resolve conflicting observations in the literature on glutamine synthetase following ischemia reperfusion, bringing together an overview of glutamine biochemistry and enzymatic studies on glutamine synthetase to suggest possible neuroprotective therapies. Gary Gibson and colleagues review some of the mitochondrial response mechanisms supporting protein signaling and how these change in Alzheimer disease.

Glutathione is yet another molecule with an intriguing biochemical cycle between astrocytes and neurons. With much yet to be determined about the many roles it plays, Ralf Dringen has contributed another great article on the tripeptide and its detoxifying activities.

Gerry's work on lactate and other metabolite spreading lead him more recently to finding ways to measure the removal of metabolites from the brain via the perivascularlymphatic pathway. This work is reflected in a number of papers in this issue; Jessen [11] have written a beginner's guide to the glymphatic system, describing its structure and functions and how it may fail in various brain diseases. The important role of aquaporin- 4 in the perivascular astrocytic endfeet is also highlighted in an up-to date synthesis of AQP-4's role in potassium clearance and edema formation [12] and the role of catecholamines volume transmission modulation of fundamental functions, including bioenergetics and the glymphatic system is also reviewed [13].

Gerry's work included a number of animal models of inborn errors of metabolism, including hepatic encephalopathy, experimental diabetes and phenylketonuria, a disorder in which the underlying reasons for neurological and cognitive impairment are still unclear. In a major undertaking, the contribution of magnetic resonance spectroscopy to study of inborn errors of metabolism and diabetes is reviewed by In-Young Choi et al. In the final section of this issue, the magnetic resonance spectroscopy and imaging come to the fore, with astrocytic swelling examined using functional diffusion weighted imaging investigate appetite regulation in the hypothalamus, contributed by Sebastian Cerdan and colleagues, and a longitudinal investigation of neurochemical changes in a mouse model of prion disease, contributed from Lausanne by Cristina Cubaldu.
All in all, a broad scope of neurochemistry and the role of astrocytes are covered in this issue which we hope gives the reader some idea of the scope of work covered by Gerry in his career. The Guest editors of this issue, Ursula Sonnewald and Caroline Rae, would like to extend their gratitude to everyone who contributed to this issue, and also to Gerry Dienel, whose ideas and input were invaluable in bringing the issue together. Gerry is a truly valued colleague and a classic neurochemist, with a depth of skill and knowledge that continues to be an inspiration to us all. We look forward to more contributions from him and from Nancy and we wish them both all the best for their future.

\section{References}

1. Teschemacher AG, Gourine AV, Kasparov S (2015) A role for astrocytes in sensing the brain microenvironment and neurometabolic integration. Neurochemical Res 40:2386-2393

2. Winkler U, Hirrlinger J (2015) Crosstalk of signaling and metabolism mediated by the NAD+/NADH redox state in brain cells. Neurochemical Res 40:2394-2401

3. Šerý O, Sultana N, Kashem M, Pow D, Balcar V (2015) GLAST but not least-distribution, function, genetics and epigenetics of 1-Glutamate transport in brain-focus on GLAST/EAAT1. Neurochemical Res 40:2461-2472

4. Öz G, DiNuzzo M, Kumar A, Moheet A, Seaquist E (2015) Revisiting glycogen content in the human brain. Neurochemical Res 40:2473-2481

5. Cruz NF, Dienel GA (2002) High glycogen levels in brains of rats with minimal environmental stimulation; implications for metabolic contributions of working astrocytes. J Cereb Blood Flow Metab 22:1476-1489

6. Box GEP, Draper NR (1987) Empirical model building and response surfaces. Wiley, New York

7. Tiret B, Shestov A, Valette J, Henry P-G (2015) Metabolic modeling of dynamic 13C NMR isotopomer data in the brain in vivo: fast screening of metabolic models using automated generation of differential equations. Neurochemical Res 40:2482-2492

8. Dienel GA, Cruz NF (2015) Contributions of glycogen to astrocytic energetics during brain activation. Metabolic Brain Dis 30:281-298

9. Lange S, Winkler U, Andresen L, Byhrø M, Waagepetersen H, Hirrlinger J, Bak L (2015) Dynamic changes in cytosolic ATP levels in cultured glutamatergic neurons during NMDA-induced synaptic activity supported by glucose or lactate. Neurochemical Res 40:2517-2526

10. Dienel GA (1984) Regional accumulation of calcium in postischemic rat brain. J Neurochem 43:913-925

11. Jessen N, Munk A, Lundgaard I, Nedergaard M (2015) The glymphatic system: a beginner's guide. Neurochemical Res 40: 2583-2599

12. Assentoft M, Larsen B, MacAulay N (2015) Regulation and function of AQP4 in the central nervous system. Neurochemical Res 40:2615-2627

13. Fuxe K, Agnati L, Marcoli M, Borroto-Escuela D (2015) Volume transmission in central dopamine and noradrenaline neurons and its astroglial targets. Neurochemical Res 40:2600-2614 